\title{
Whooping cough and unrecognised postperinatal mortality
}

\author{
A NICOLL* AND A GARDNER† \\ ${ }^{*}$ Department of Child Health, Queen's Medical Centre, Nottingham, and †Division of Medical Statistics, \\ London School of Hygiene and Tropical Medicine.
}

SUMMARY Trends in postperinatal infant mortality from respiratory causes and the sudden infant death syndrome in England and Wales for 1968 to 1984 were examined. These were compared by time series analysis with changes in the incidence of specific infective diseases and organisms for the same period. Discontinuity was found in association with the occurrence of whooping cough between 1977 and 1982. Associations with the general incidence of respiratory infections and other specific organisms were less evident. An estimate of excess mortality is 460 to 700 deaths, a substantial increase over the certified mortality from whooping cough.

Following concern over the safety of whooping cough vaccine in the middle 1970 s, the percentage of children in England and Wales who were fully immunised by the age of 2 years halved. Subseuently epidemics (which occur at about four year intervals) increased in size; fig 1a shows notifications for infants aged $0-1$ years. ${ }^{1} 2$ Mortality remained low, only 54 infants having whooping cough as their primary cause of death between 1977 and 1983, compared with 65 in the preceding seven years, ${ }^{3}$ suggesting that whooping cough was becoming a less serious disease. ${ }^{4}$ Equally likely was that the true mortality from the disease was unrecognised. The mortality is highest among younger patients, in whom the disease can present without the typical whoop or paroxysmal coughing. ${ }^{5}$ In Nottingham during the epidemic 1985-1987 Bordetella pertussis caused one near-miss cot death and a fatal respiratory infection in which the diagnosis would easily have been missed from the clinical signs alone. Similar cases have been reported elsewhere. ${ }^{6}$ Both respiratory infection ${ }^{5}$ and sudden infant death syndrome (SIDS) have been certified causes of death in whooping cough fatalities. ${ }^{7}$ Many cases of SIDS have signs of a respiratory illness compatible with early whooping cough. ${ }^{8}$ The terminal apnoea of whooping cough could be indistinguishable from a sudden infant death at home, ${ }^{9}$ but are these covert deaths from whooping cough a measurable cause of infant mortality? If they are we would expect discontinuity in infant mortality to be associated with an increased incidence of whooping cough.

\section{Methods}

To look for temporal associations in infant mortality and the incidence of the disease and of other organisms we used time series analysis. By use of different spans and weighting it was possible to investigate long term trends and short term events. ${ }^{10}$

\section{CHANGES IN INFANT MORTALITY}

Data were obtained from death certificates for infant deaths in England and Wales from 1968 to 1984. The study allowed for two factors that could have influenced the reporting. Firstly, after 1970 infant respiratory deaths were increasingly recorded as being due to SIDS; figures for the diagnoses reflect the trend in reclassification (fig 2). Secondly, the recording of unexpected infant deaths is not standard on death certificates and includes respiratory deaths and asphyxial deaths defined as a death thought to be due to accidental mechanical smothering-for example, in the bed clothes or by inhalation of vomit), as well as SIDS itself. ${ }^{811}$ To allow for both factors an estimate of the true trend (fig 2) was derived by adding all the deaths in a particular quarter that were ascribed to respiratory causes (International Classification of Diseases (ICD) codes 460-519), asphyxial causes (ICD codes E911 and E913) and sudden infant death (ICD code 795 until 1978, thereafter code 798). Quarterly rates (referred to as combined mortality) were calculated by dividing these totals by the number of live births in the preceding quarter. 
42 Nicoll and Gardner

(a)

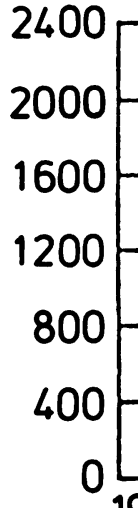

Notifications of pertussis age $0-1$ year

Fig 1a Quarterly pertussis notifications to OPCS for children aged 0-1 years in England and Wales (1968-85).

(b) Combined mortality (three year moving average)

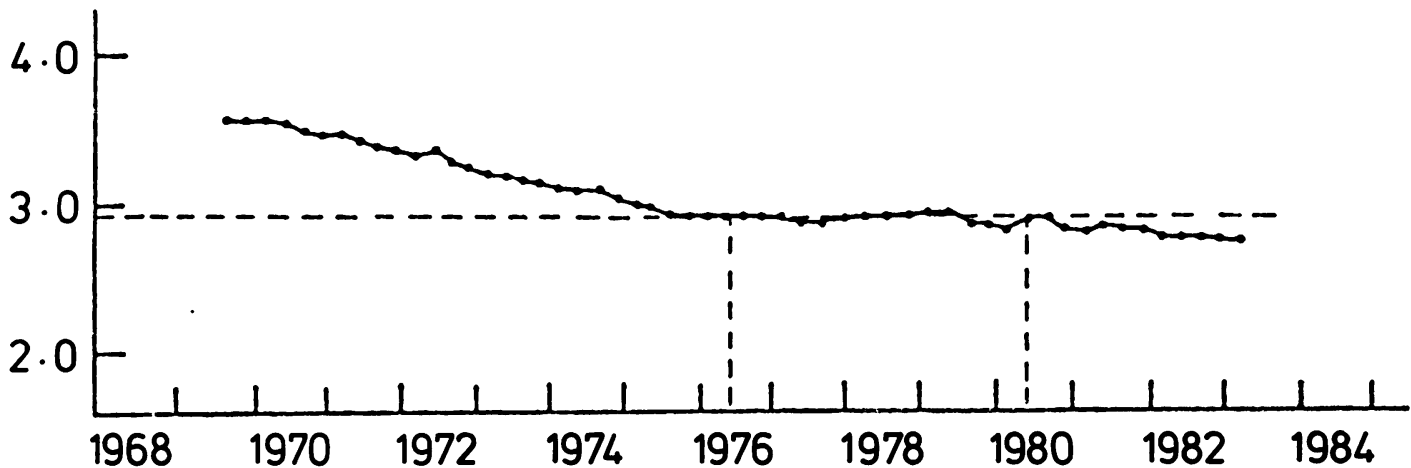

Fig 1b Three year span moving average mortality for combined respiratory, asphyxial, and sudden infant deaths in

England and Wales plotted quarterly for the period 1970-83. Dotted vertical lines indicate period decline in mortality halted and resumed.

\section{(C)}

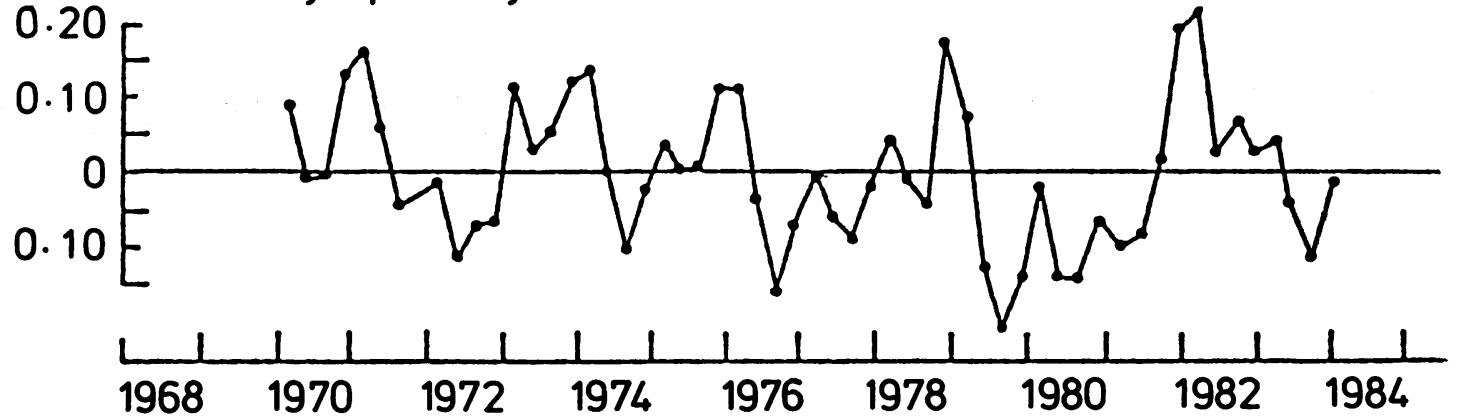

Mortality (quarterly residuals)

Fig 1c Quarterly residuals of combined mortality (1970-1983). 


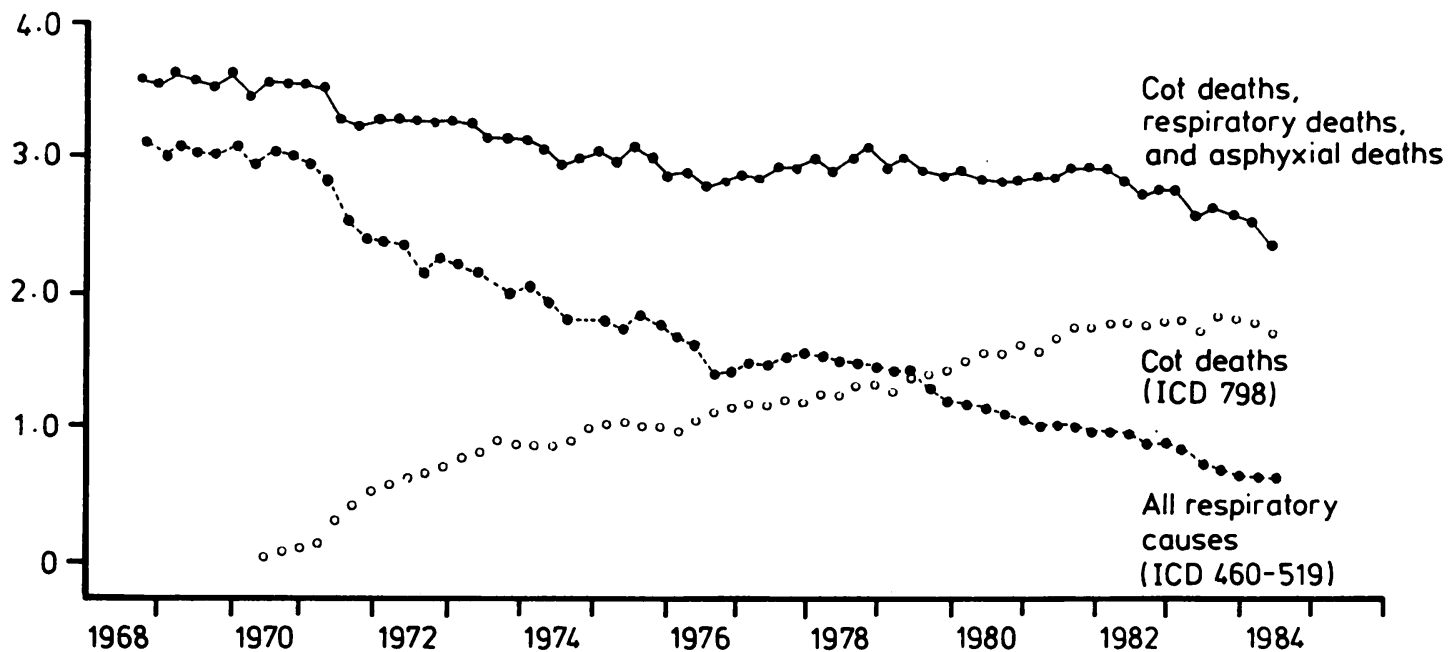

Fig 2 Moving annual mortality plotted quarterly for infant deaths from all respiratory causes, sudden infant deaths, and all respiratory causes, sudden infant deaths plus asphyxial deaths (OPCS data).

This denominator was chosen because 3 months is the peak age of risk for infants dying of respiratory causes and SIDS, and its use is recommended when calculating infant mortality from unexpected death. ${ }^{11}$ Large seasonal variations in deaths from respiratory causes and SIDS are well known and to look for associations such seasonal swings must be removed. ${ }^{11}$ This was done by time series analysis using graphs of moving averages-for example, in fig 2 each point represents the mean of four quarterly rates. To see longer term trends a three year rolling average was calculated (fig 1b). Each point was calculated by taking the mean quarterly rate (without weighting) for a three year period plotted at the midpoint. The next point is calculated by moving the three year span forward one quarter, and so on. The same figures for combined mortalities were used to calculate short terms events; short term quarterly rates were calculated using a span of nine and peak weighting of 256 by making eight summations in series of adjacent pairs of the original rates. ${ }^{10}$ Residuals (fig 1c) were calculated by subtracting each short term rate from the appropriate three year average. A positive residual represents a quarter when combined mortality was above the three year norm, a negative residual the converse. No other time series analysis was performed.

CHANGES IN THE INCIDENCE OF DISEASES AND ORGANISMS

Trends in the incidence of $B$ pertussis, other potentially lethal infectious organisms, and respira- tory disease in general were examined. Data were available from the three sources: disease notifications to the Office of Populations, Censuses and Surveys (OPCS) ${ }^{1}$ the Royal College of General Practitioners (RCGP) weekly returns, ${ }^{12}$ and organism reports to the Public Health Laboratory Service (PHLS). ${ }^{2}$

Notifications of whooping cough to the OPCS for children aged $0-1$ year were plotted quarterly for the period 1968 to 1985 (fig 1a). ${ }^{1}$ All other diseases and organisms were plotted over the same period. Quarterly notifications of whooping cough at all ages from the RCGP, and reports of $B$ pertussis from the PHLS were treated similarly (data not shown).

The quarterly incidences of all respiratory diseases with a possible infective cause (common cold, influenza-like illness, sore throat and tonsillitis, laryngitis and tracheitis, epidemic influenza, pneumonia and pneumonitis, acute bronchitis, pleurisy, and asthma) for all ages reported by the RCGP were plotted. These data were only made age specific in 1981, and then only in broad bands-that is, $0-4$ years. To remove annual variation these rates were treated in a manner identical with the combined mortalities to show long term (fig 3a) and short term (fig $3 b$ ) changes. Quarterly rates for the same disease categories for patients aged $0-4$ years were plotted for 1981 onwards but could not be subjected to time series analysis because of the limited period.

Quarterly reports for all ages for infective organisms identified by the PHLS were studied. 


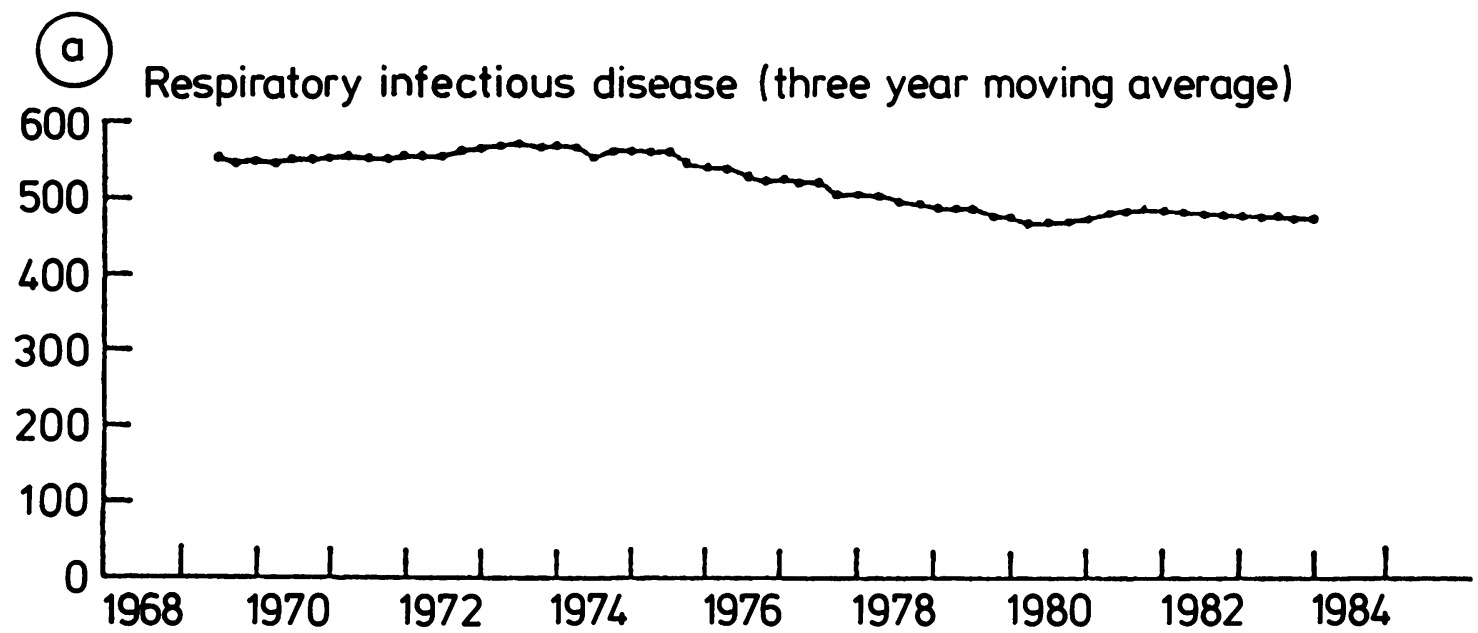

Fig 3a Three year moving average of incidence of infective respiratory disease for all ages from the RCGP plotted quarterly for the period 1970-84.

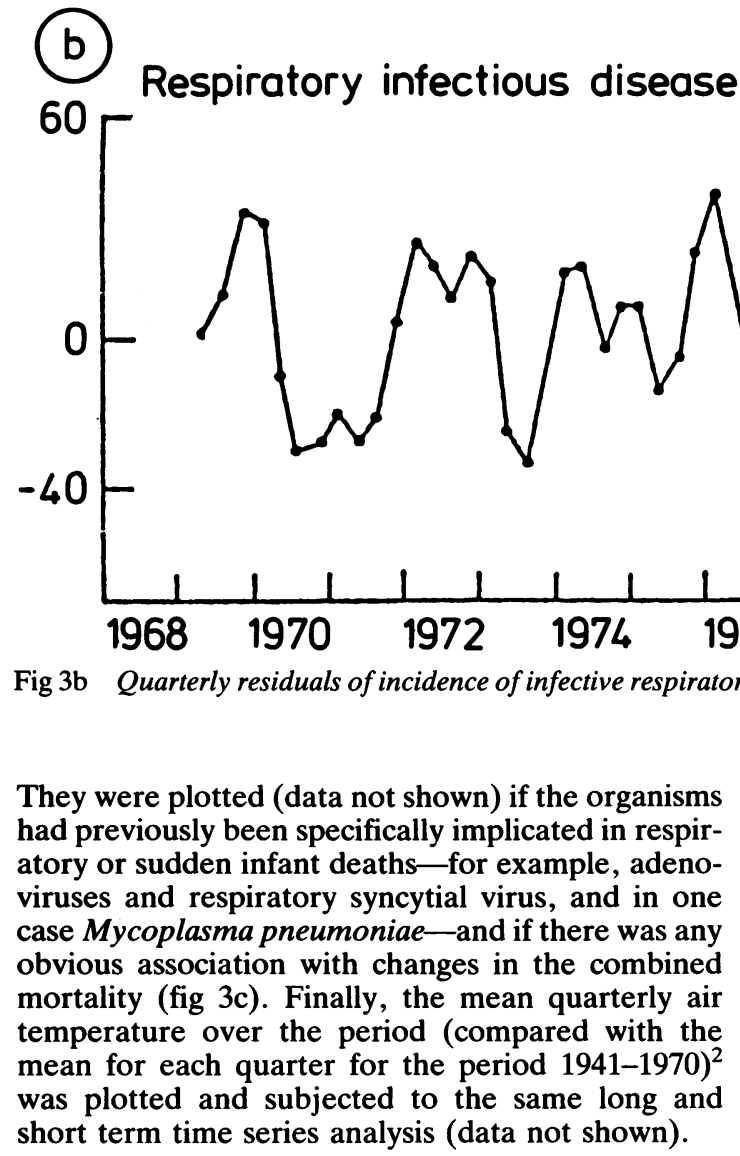

\section{Results}

The trends in the notifications of whooping cough (fig 1a) showed not only that there were epidemics in 1977/9 and 1981/2 but that notifications remained high in between - that is, there was an extended epidemic from 1977 to 1982 . This was confirmed by the RGCP and PHLS reports. There was a temporal association between long term changes in the combined mortality and the incidence of whooping cough (figs 1a and 1b), though the decline in 


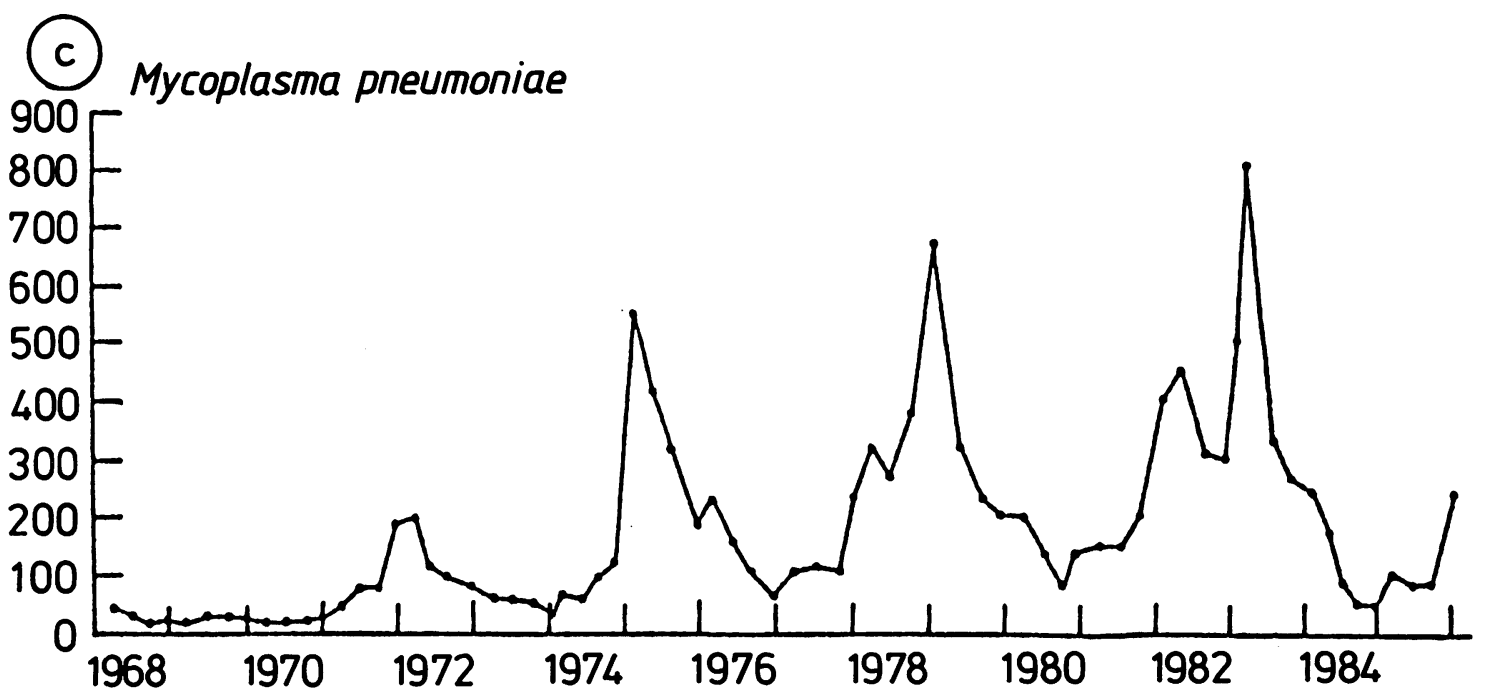

Fig 3c Quarterly identification of M pneumoniae by the PHLS (1968-1985).

mortality preceded the end of the extended epidemic. The rate showed a gradual decline from 1968 to 1976 followed by a flattening of the curve until mid 1980; the downward trend then resumed at a reduced rate. The one year moving average (fig 2) showed a similar decline, but with more short term events superimposed. The differences in rates could not be compared directly from fig $1 b$ because consecutive points were not independent, thus a graph of every fourth point of the one year average was considered (not shown). This divided into three clearly defined periods: mid 1969 to mid 1976, mid 1977 to mid 1980, and mid 1981 to mid 1984. The slopes for the three were compared using linear regression analysis. The regression coefficients were: $b=-0 \cdot 109, b=-0 \cdot 004$, and $b=-0.062$, respectively. The slopes for the first and second periods were significantly different $(p<0 \cdot 02)$. The differences were not significant when the earlier periods were compared with the third, which may reflect the limited time scale of the latter. Short term mortality after 1976 also had a close temporal correlation with epidemics of whooping cough (figs 1a and 1c), though such events also occurred before 1976.

Due to regular seasonal variations the rates of respiratory illness reported by the RCGP before time series analysis did not show any obvious trends for either all age or $0-4$ years plots. The long term trend (fig 3a) showed an overall decline but no association with the flattening of the combined mortality curve, and the quarterly residuals peaked in association with the epidemics of whooping cough (fig 3b).

With the exception of $M$ pneumoniae in the short term (fig 3c), there was no association between organisms reported by the PHLS and the air temperature, and changes in the combined mortality.

A calculation of the excess infant mortality was made from yearly figures using the flattening from mid 1977 to mid 1980 in the graph calculated from the one year average. The population was taken as the live births in the 12 month period starting three months before each year. Two rates of decline were applied: that of mid 1969 to mid 1976 (high excess risk) and that of mid 1981 to mid 1984 (low excess risk). This gave figures (corrected to the nearest 10) of 700 (high excess risk) and 460 (low excess risk). In the same period the number of certified deaths due to whooping cough was 32 , giving factor differences of 22 and 14.

\section{Discussion}

The discontinuity in long term mortality trends indicates an excess of infant deaths in certain categories during the period 1976 to 1980 . We have examined the hypothesis that this was substantially due to undiagnosed whooping cough, and that we may be underestimating its mortality. The long term association between the incidence of whooping cough and the combined mortality supports the hypothesis. (figs $1 \mathrm{a}$ and $1 \mathrm{~b}$ ). The early decline of mortality in mid 1980 was explained by the steep 
decline in shorter term rates after 1981 (fig 2). There was also a close temporal relation between short term combined mortality and the epidemics of 1977-9 and 1981-3 (figs 1a and 1c). Variations in mortality also occurred, however, before 1976 when epidemics were small and unlikely to make detectable impacts. Hence the previous fluctuations must have been due to unknown factors that may still have been present after 1976. There was also an association between the combined mortality and the fluctuations in the incidence of respiratory diseases in the community (figs $1 \mathrm{c}$ and $3 \mathrm{a}$ ). This finding must be interpreted with caution as the data from the RCGP may not be representative of the community as a whole. ${ }^{12}$ If it is true, then either the short term changes in combined mortality resulted from broad fluctuations in the incidence of all respiratory diseases that happened to coincide with epidemics of whooping cough, or the fluctuations in the incidence of respiratory diseases represented covert infection with $B$ pertussis in adults in whom the presentation can be atypical as it can in infants ${ }^{5}{ }^{13}$; as there are no associations between the long term combined mortality and the rates of respiratory diseases (figs $1 b$ and 3a) the latter seems more likely.

Time series analysis is an indirect measure of cause and effect; it can only suggest associations and support hypotheses. It is especially vulnerable to confounding phenomena as it is unable to exclude the possibility of an alternative factor showing a better temporal association. ${ }^{10}$ There is also a danger of applying many calculations and selecting those which fit a hypothesis, which is why such manipulations were limited in this study. Is there therefore any further support for the hypothesis? The unusual presentation of whooping cough in the younger patients is additional evidence; this phenomenon has also been reported clinically ${ }^{6}$ and there is a pathophysiological mechanism. ${ }^{9}$ Cherry ${ }^{14}$ and Miller et al, ${ }^{15}$ using indirect methods that were different from ours have shown similar epidemiological patterns and similar excesses in mortality, and in 1931 Stallybrass wrote: 'During the prevalence of whooping cough the rise in the respiratory mortality (bronchitis and pneumonia) under five years of age is often considerably greater than the number of deaths certified as from whooping cough. ${ }^{16}$

The number and type of organisms reported to the PHLS do not reflect the true incidence of disease in the community so we must not dismiss entirely the role of other organisms. Neither can we conclude that such organisms do not cause individual deaths, merely that they do not seem to affect changes in incidence or are too rare to impinge on a population study. The only organism that showed any association was $M$ pneumoniae and it did not show any short term association before 1976 (figs 1c and 3c), or any long term association. It is also unlikely to cause substantial covert infant mortality; it is uncommon in pre-school children, rarely causes epidemics, and the severity increases with age. ${ }^{17}$

We do not wish to imply that whooping cough is the major cause of SIDS. Even if all the excess deaths were due to whooping cough and half appeared as SIDS it could only account for $0.7 \%$ to $11 \%$ of deaths attributed to SIDS from 1977 to 1980 . It is of interest, however, that when notifications of whooping cough finally returned to their usual interepidemic low in 1984 (fig 1a), notifications of SIDS also declined (fig 2). ${ }^{18}$

The implications of these findings are considerable. Professional and public complacency about whooping cough is one of the factors contributing to poor acceptance of vaccination. The rare dangers associated with immunisation have received a great deal of publicity; a more accurate measurement of the disease's mortality is required so that doctors and nurses can make balanced decisions over the risks and benefits of protecting children.

We thank the Office of Population Censuses and Surveys, the Royal College of General Practitioners Weekly Returns Service, and the Public Health Laboratory Service for providing the data, and Mrs Gillian McNab for preparing the manuscript.

\section{References}

1 Office of Population Censuses and Surveys. Comunicable disease statistics. Series MB2. London: HMSO, 1968-1985.

2 Office of Population Censuses and Surveys. Quarterly infectious diseases monitors. Series MB2. London: HMSO, 1968-1985.

3 Office of Population Censuses and Surveys. Childhood and maternity mortality. Series DH3. London: HMSO, 1968-1985.

4 Stewart GT. Whooping cough and pertussis vaccine. Br Med J 1983;287:287-9.

5 Christie AB. Infectious diseases. Edinburgh: Churchill Livingstone, 1980:659-82.

6 Parks Y, Sunderland R. Sudden febrile infant deaths. Lancet 1986;i:764-5.

7 Royal College of General Practitioners, Swansea Research Unit. Effect of a low pertussis vaccination uptake on a large community. Br Med $J$ 1981;282:23-6.

${ }^{8}$ Knowelden J, Keeling J, Nicoll JP. A multi-centre study of postneonatal mortality. Sheffield: Medical Care Research Unit, University of Sheffield, 1984.

9 Southall DP, Thomas MG, Lamber HP. Prolonged expiratory apnoea and severe hypoxaemia in infants with pertussis. Arch Dis Child (in press).

${ }^{10}$ Chatfield C. The analysis of time series: theory and practice. London: Chapman and Hall, 1975.

"Carpenter RG, Gardner A. Variations in unexpected infant death rates relating to age, sex and season. In: Office of Population Censuses and Surveys. Studies in sudden infant deaths. London: HMSO, 1982. (Studies on Medical and Population Subjects. No. 45.) 
12 Fleming DM, Crombie DL. The incidence of common infectious diseases: the weekly returns service of the R.C.G.P. Health Trends 1985;17:13-6.

${ }^{13}$ Broome CV, Preblud JR, Bruner B, et al. Epidemiology of pertussis, Atlanta 1977. J Pediatr 1981;98:362-7.

14 Cherry JD. The epidemiology of pertussis and pertussis immunisation in the United Kingdom and the United States: a comparative study. Curr Probl Pediatr 1984;14:1-78.

${ }^{15}$ Miller D, Wadsworth J, Diamond J, Ross E. Pertussis vaccine and whooping cough as risk factors in acute neurological illness and death in young children. Dev Biol Stand 1985;61:389-94.
${ }^{16}$ Stallybrass CO. The principle of epidemiology. London: Routledge, Kegan \& Paul, 1931:280.

17 Broughton RA. Infections due to mycoplasma pneumonia in childhood. Pediatr Infect Dis 1986;5:71-85.

18 Office of Population Censuses and Surveys. Sudden infant death syndrome 1983-1984. Series DH3 85/5. London: HMSO, 1985.

Correspondence to Dr A Nicoll, Department of Child Health, Queen's Medical Centre, Nottingham NG7 2UH.

Received 13 August 1987 